\title{
Enhancing timetable planning with stochastic dwell time modelling
}

\author{
G. Longo \& G. Medeossi \\ Dept. of Civil and Environmental Engineering, University of Trieste, Italy
}

\begin{abstract}
Estimating stop time is a critical task for timetable planning, especially since actual dwell time shows a significant variability that could hinder service reliability. Therefore its importance has increased as railways seek to maximize infrastructure utilization while improving the quality standards, including reliability.

A significant amount of scientific work focussing on the estimation of dwell time within different transit systems can be found in literature. However, it is mostly focused on aspects such as, for example, the time required for each passenger to board depending on the number of steps, while in very few reports the applicability of the models and their practical relevance are described.

This study presents an approach for estimating the stop time distribution of train services and using them in simulation or timetable planning tools. Based on conventional parameters on the one hand and on track occupancy data collected by train describers on the other, the models include an algorithm to derive the stop time from the track occupancy data and a multi-parametric dwell time estimation.

When past track occupancy data is available, the parameters are estimated: the calibrated model can be used as input to plan or simulate future scenarios considering, for example, new rolling stock or different service patterns.

The results of model calibration and its use in a test network around Venice are described in the last part of the paper.

Keywords: stochastic simulation, railway planning, dwell time, train performance.
\end{abstract}




\section{Introduction}

Achieving the highest accuracy in timetables is paramount for public transport operators, since it allows a precise estimation of vehicle and crew scheduling as well as improves the quality level perceived by passengers.

The importance of accuracy in planning is especially relevant in railway systems, where a proper timetable allows operating a higher number of trains without significant infrastructure investments.

Stochastic micro-simulators can reproduce most processes involved in rail traffic and comprehend not only its deterministic aspects, but also human factors. This is particularly relevant in order to simulate traffic under realistic conditions, considering variability at border, various driving styles and stop times. All these parameters have to be calibrated using real-world collected data for single trains or train families, considering their different behaviour in the network and at its border.

Stochastic micro-simulation uses very detailed data as input. They include the deterministic data conventionally used to estimate train motion in timetable planning [1]:

- $\quad$ The infrastructure model

- $\quad$ The description of the rolling stock

- $\quad$ The planned timetable

and some stochastic parameters that allow reproducing the real traffic variability:

- $\quad$ The variability when entering the simulated network

- A set of parameters that reproduce the behaviour of drivers

- $\quad$ The stop time.

The block diagram in Figure 1 shows the input and output of stochastic micro-simulation.

STOCHASTIC INPUT

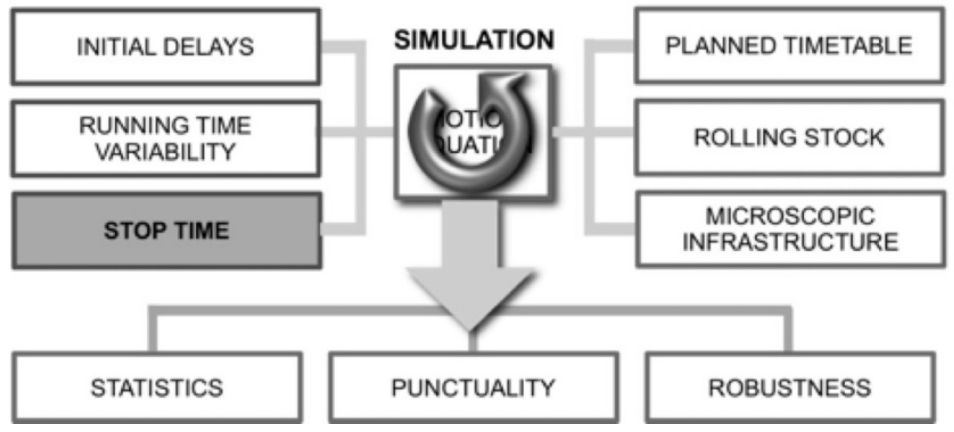

Figure 1: Deterministic and stochastic input and output of stochastic micro-simulation. 
Microscopic infrastructure models offer a realistic representation of the deterministic constraints on train movements that leaves only minor errors. Tests carried out in multiple countries proved the accuracy of commercial tools like OpenTrack and RailSys and they are now used worldwide.

A very accurate, reliable method for calibrating the driving styles of train drivers on the basis of on-board collected data has been presented recently by the authors [2]. It allows encapsulating in a parameter set all differences between train motion represented by the conventional motion equation and the real behaviour of trains.

The departure variability of each train entering in the simulated area is derived by conventional data collected at infrastructure level by infrastructure managers using track circuits.

A blank spot still remains concerning the representation of stop time variability. In commercial tools it is simply represented by a departure delay function defined by user, while in literature a more accurate stochastic model is hardly found.

Significant amount of scientific work focussing on the estimation of dwell time with in different transit systems can be found in literature. However, it is mostly focused on its deterministic aspects, such as for example the time required for each passenger to board depending on the number of steps, while a model which includes both deterministic and stochastic elements is hardly found.

The study presents an approach for estimating the stop time distribution of train services. Based on conventional parameters on one hand and on track occupancy data collected by train describers on the other, the models includes an algorithm to derive the stop time from the track occupancy data and a multiparametric dwell time estimation.

When past track occupancy data is available, the parameters are estimated: the calibrated model can be used as input to plan or simulate future scenario considering for example new rolling stock or different service patterns.

\section{Literature review}

While a simple model that allows integrating an accurate estimation of dwell times in micro-simulation of conventional railways apparently has not been developed yet, a broad literature exists, which deals with the dwell times of rail and transit services.

The dynamics of passenger interchange in public transport systems were first broadly investigated by Weidmann [3]. Following the approach proposed by Tuna [4], Rueger [5], analysed dwell times at multiple stations in Switzerland, Germany and Austria, defining an empiric model based on the characteristics of the passenger, the train and the luggage.

In a wide-spectrum study to improve schedule reliability on Zurich S-Bahn Nash et al. [8] analysed the barding/alighting process focussing on the congested station of Stadelhofen. The study started with a detailed analysis of the physical layout of stations, especially considering the position of stairs and the roof coverage, pointing out a relationship between them and the overcrowding of 
some coaches that increased the dwell time. Second, the S-Bahn rolling stock for passenger boarding and alighting problems was evaluated. The third step in the analysis was observation of passenger boarding and alighting at the five stations during the morning peak period. This data was used to help identify boarding/alighting problems and ultimately to develop standard station dwell time curves.

This work was further improved by the same authors [8], leading to a dwell time estimation model that allows planners to predict station dwell time (mean and spreading) by entering the following input parameters: vehicle type (number, position, width and level of doorways), station infrastructure (platform level) and demand (number and distribution of boarding and alighting passengers). Despite of the extensive validation tests and of the first promising results, the model apparently has not been further improved nor applied in practice.

More recently Wang et al. [10] developed an algorithm for the calibration of urban rail simulation models. The model algorithm implements the model developed by Puong [11] to represent dwell time using four parameters: a constant, a boarding time per passenger $\alpha$, an alighting time per passenger $\beta$, and crowding factor $\gamma$. Standard values are used as initial values and are calibrated during the calibration.

The goal of this work is to develop a model that starts with the model proposed by Wang, and first extends it to a European context considering also the variable used by Buchmueller. A significant drawback of such a model would be the amount of data required for such a model and therefore, the defacto inapplicability to large contexts. To solve it, the results were generalised and the model improved to enable the use of simple data automatically collected at stations using track circuits and currently used in micro-simulation to define the initial delay distributions.

\subsection{A dwell time model for railways}

A train's station dwell time is determined by the combination of the passenger boarding/alighting process, the door control system processes, and actions taken by the train driver and infrastructure operator $[1,2]$. There are two main parts of the station dwell process: passenger service time and train dispatching time. In order to separate the influences of the different actors the station dwell process was first divided as proposed by Buchmueller into the first 5 sub-processes shown in Table 1. It was also necessary to separate between late and early arriving trains, since in the second case the sixth element must be included.

In fact, on early running trains, when the passenger service has finished the driver waits (WA) before closing the doors and departing. Although the train is ready for a punctual, precise departure, it might happen that due to passengers showing up at the last second or due to poor coordination between the crew the doors will be closed seconds late. This slight delay is called Departure Impreciseness (DI). Figure 2 shows the total dwell time of late-running trains (left) and the departure delay of early-arriving trains (right), for all Regional services in Monfalcone in January and February 2012. Without separating the two cases, the stop time distribution would be increased by these longer stops. 
Table 1: $\quad$ Dwell time sub-processes.

\begin{tabular}{|l|l|l|l|}
\hline Sub-process & Location & Process Begins & Process Ends \\
\hline Door-unblocking (DU) & 1 Doorway & Train arrival & Begin door opening \\
\hline Door opening (DO) & 1 Doorway & Begin door opening & $\begin{array}{l}\text { First passenger through } \\
\text { the doorway }\end{array}$ \\
\hline Boarding/alighting (BA) & 1 Doorway & $\begin{array}{l}\text { First passenger through the } \\
\text { doorway }\end{array}$ & $\begin{array}{l}\text { Last passenger through } \\
\text { the doorway }\end{array}$ \\
\hline Door closing (DC) & 1 Doorway & $\begin{array}{l}\text { Driver activates door } \\
\text { closing }\end{array}$ & Door closed \\
\hline Train departure (TD) & Whole Train & $\begin{array}{l}\text { Last door closed } \\
\text { Train departure }\end{array}$ \\
\hline Traiting (WA) & Whole train & $\begin{array}{l}\text { Last (punctual) passenger } \\
\text { through the doorway }\end{array}$ & $\begin{array}{l}\text { Driver activates door } \\
\text { closing }\end{array}$ \\
\hline
\end{tabular}
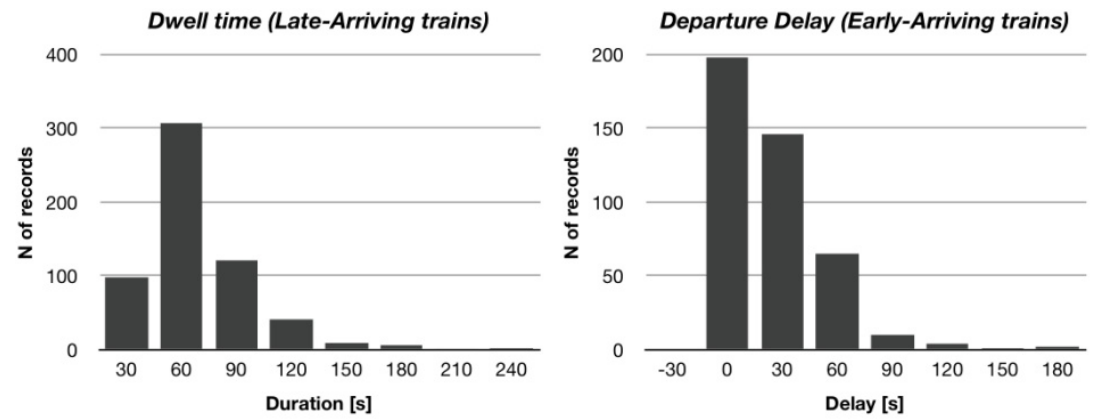

Figure 2: Total dwell time of late-running trains (left) and departure delay of early-arriving trains (right).

\section{A dwell time model to be used in simulation}

As stated before, the goal of this research project was to develop a dwell time model that, after an initial accurate calibration phase could be easily generalized and applied to different networks and used in practice. Therefore except for the initial calibration, the model was supposed to use only the track circuit data.

However, differently than other possible (but less common) inputs, these data do not contain an explicit measure of the dwell time, since they provide the instant in which a train enters the station track circuit and the instant in which it leaves it. The arriving - dwell - departure process of a train on a track circuit is represented in Figure 3. The speed profile of the train, including its mean deceleration and acceleration is shown on the top; in the middle, the station track circuit is highlighted, while at the bottom the occupation time $(O)$ is decomposed in occupation before the arrival $(A O)$, stop time and occupation after the departure of the train $(D O)$. 


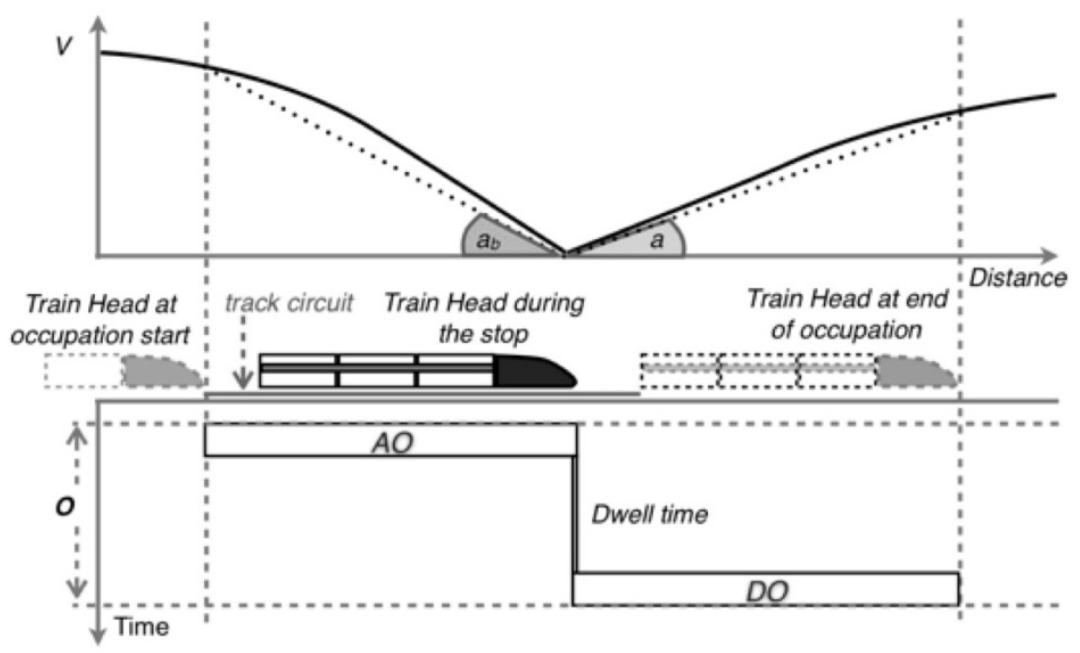

Figure 3: $\quad$ Speed profile, track circuit occupation and stop time.

As a result, the first step to be performed is to remove the occupation time before the train stops and after its departure ( $A O$ and $D O$ in Figure 3).

This filtering can be performed in a quite simple way once the length and position of the track circuit with respect to the stopping position $(L$ and $P)$ is known. This information is included in the interlocking plans of station, and therefore available when preparing a simulation model.

The second set of parameters that must be considered represents the dynamic behaviour of the train when braking $\left(a_{b}\right)$ and accelerating $(a)$ and its length $L_{T}$. Since most stations have very low gradients and trains on the station track circuit run with very low speed (since they are stopping) it is possible to consider mean acceleration and deceleration values for each trainset.

Once these parameters are estimated (they are normally already included in simulation models), the estimation of the dwell time on the basis of track circuit occupation time is performed very simply as:

$$
D=O-A O-D O
$$

where

$$
\begin{gathered}
A O=\sqrt{\frac{2(L-P)}{a_{b}}} \\
D O=\sqrt{\frac{2\left(L_{T}+P\right)}{a}}
\end{gathered}
$$

The result is the total dwell time described in section 2. Since the occupation data include the absolute (or relative) time of arrival and departure, it is possible to separate between early and late arriving trains. 
Since the dwell time of early arrival trains can include the waiting time and the departure impreciseness, they cannot be used to estimate the other subprocesses. In fact, it appears impossible to separate them since the waiting time is unknown. Therefore the early (and punctual) arriving trains are filtered out and stored separately.

Even considering only the delayed trains, the dwell time measured using the occupation time cannot be decomposed in its sub-processes. However, considering them separately:

a) The door unblocking (DU) and door opening (DO) are only few seconds long; moreover the tests carried out during this study confirm the results extensive obtained by Buchmueller in Switzerland showing that their variability is extremely low when considering a single type of trainset. As a result, in this work the DU and DO were considered as fixed for each trainset, since the error introduced in the model ( \pm 3 seconds) is irrelevant in stochastic simulation.

b) Similarly, The door closing (DC) sub-process contains a nearly deterministic, trainset-specific time similar to the DU and DO. Differently than Buchmueller et al. [9], who considered each single door automatically closing after a specific time period with no passengers boarding and alighting, in this model the door closing sub-process contains only the time required for closing and blocking the last door. Despite of a slightly higher variability, compared to the DU and DO, also the DC was considered as deterministic in the model.

c) The train departure (TD) sub-process considers the whole train and consists of the time period between the moment when the last train door has closed and the train departure. It is the time required by the driver to release the brakes, activate traction and let the train move. The TD is specific for each trainset and depends on its technical equipment; normally on long trains and formed by coaches and locomotive it appears significantly longer than on multiple units.

d) The boarding/alighting (BA) sub-process is the time period of passenger flows at the doorway. In order to obtain comparable values the BA subprocess was evaluated based on mean passenger flow rates $F$ (the ratio between the number of passengers and the BA sub-process time) calculated using the measured data. If these rates could be considered as function of the physical characteristics of the doorway of a trainset (according to Rueger [5] and Puong [11]), the BA would be obtained simply dividing the number of passengers in the longest queue along the train by the flow rates F. The method used to estimate F is described in Section 2.3.

Simplifying the conventional dwell time model as described, the process could be represented by a number of deterministic parameters fixed for a station and a trainset, and by a stochastic one, that is the number of passengers boarding and alighting (Table 2). A second stochastic element allows considering some influences on the flow rates due to the luggage or to the age according to Tuna [4] and Rueger [5]. 
Table 2: $\quad$ Deterministic and stochastic parameters in the dwell time model.

\begin{tabular}{|c|c|c|c|c|}
\hline \multicolumn{2}{|c|}{ Parameter } & Description & Function of & Det/Stoc \\
\hline \multicolumn{2}{|c|}{$\mathrm{DU}$} & Door unblocking & Trainset (Door system) & Deterministic \\
\hline \multicolumn{2}{|c|}{ DO } & Door opening & Trainset (Door system) & Deterministic \\
\hline \multicolumn{2}{|c|}{$\mathrm{DC}$} & Door closing & Trainset (Door system) & Deterministic \\
\hline \multicolumn{2}{|c|}{ TD } & Train Departure & Trainset (Kind, Equipment, N. of coaches) & Deterministic \\
\hline \multirow{3}{*}{ BA } & $\mathrm{F}$ & Flow rates & Trainset (Door width, $\mathrm{N}$ of steps) & Deterministic \\
\hline & $\mathrm{P}$ & No of passengers & - & Stochastic \\
\hline & $\mathrm{L}$ & Age, Luggage, etc & Age, Luggage, etc & Stochastic \\
\hline
\end{tabular}

\subsection{Adapting the dwell time model to early running trains}

As stated before, early arriving trains normally have a longer stop time compared to the minim required by the passengers since they wait for the planned departure and, after that, they could leave with a slight delay due to an impreciseness of the crew or to late-arriving passengers. It is impossible to know a priori whether this slight delay at departure is due to these perturbations or to the normal processes: in this case the planned dwell time would be under dimensioned.

A rough separation is possible when the dwell time effectively required for the 5 "basic" sub-processes is known and therefore when it has been measured on some delayed trains, subtracting the total dwell time of an early-running train by the dwell time of delayed courses.

If the measured dwell time is higher than the corresponding time for laterunning trains, the residual door-closing delay is stored as departure impreciseness (DI); otherwise the dwell time is saved together with late running trains to obtain the dwell time distribution.

\section{First results}

The first tests have been carried out on the Trieste-Venezia line in North-Eastern Italy to estimate sub-processes that could be considered deterministic in simulation. The mean and standard deviation of these sub-processes for each trainset used on the line are shown in Table 3.

Differently from the analysis contained in the other studies considered in this work, after the boarding and alighting (BA) is completed, a quite variable waiting time (WA) was measured not only for the early running rains, but also for the delayed ones. During this time the train guard checks that no passenger is coming, and communicates the driver and the passenger that the train will depart using the whistle and eventually a flag. The duration of this sub-process appears related with the number of trailers (or elements) of the train. 
Table 3: $\quad$ Mean and standard deviation of the trainset-related sub-processes.

\begin{tabular}{|l|c|c|c|c|c|c|c|}
\hline \multirow{2}{*}{ Trainset } & \multirow{2}{*}{ Descr. } & \multicolumn{2}{|c|}{ DU+DO } & \multicolumn{2}{c|}{ DC } & \multicolumn{2}{c|}{ TD } \\
\cline { 3 - 8 } & & Mean [s] & St. Dev.[s] & Mean [s] & St. Dev. [s] & Mean [s] & St. Dev. [s] \\
\hline MDVC & $\begin{array}{c}\text { Loco + } 6 \\
\text { Coaches }\end{array}$ & 3 & 1 & 6 & 2 & 18 & 4 \\
\hline Ale801 & EMU/4 & 2 & 2 & 3 & 2 & 5 & 3 \\
\hline Ale501 & EMU/3 & 3 & 2 & 5 & 2 & 7 & 3 \\
\hline
\end{tabular}

Interestingly, the model showed relatively low departure impreciseness with about $80 \%$ records lower than 10 seconds and the other records quite dispersed: also excluding the outliers some records are higher than 30 seconds. The distributions of $W A$ for a trainset formed by a locomotive and 6 coaches and of $D I$ for all trainsets are shown in Figure 4 respectively on the right and on the left.
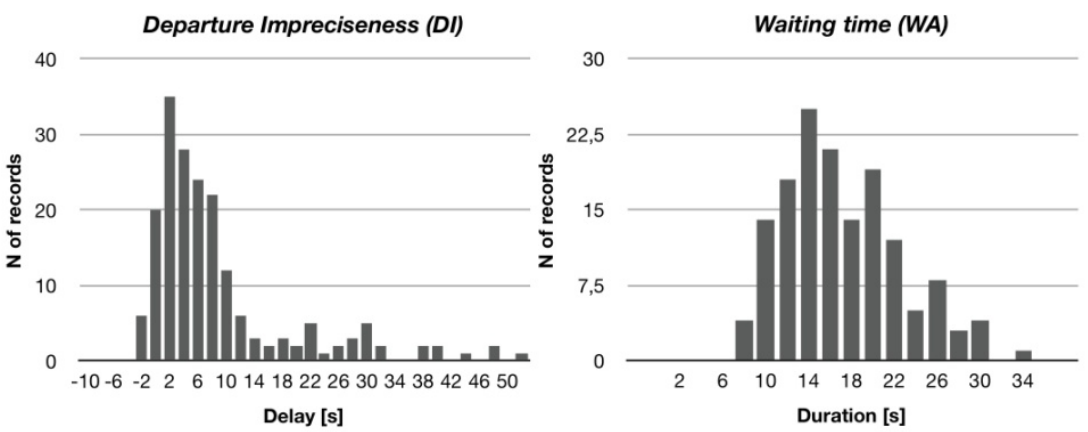

Figure 4: Departure Impreciseness (DI) of early-arriving trains (left) and Waiting Time (WA) of punctual and delayed trains.

The data collected manually at stations were insufficient to allow estimating the boarding and alighting process as a function of the number of passengers and of the flow rates. Therefore, to enable testing the model in a simulation study, the sub-process $B A$ was modelled as a stochastic distribution obtained from the track circuit data by subtracting the other sub-processes.

With this assumption the model was tested as an input for the calibration of a stochastic micro-simulation of the network. The manually collected data were used to estimate the trainset-related and deterministic sub-processes $(D U, D O$, $D C)$, as well as the stochastic ones (DI, WA). The WA was estimated for 100 days from the track circuit data by subtracting the arrival and departure 
occupation, the other sub-processes, using the mean value for the deterministic ones and a random value from the measured distribution for the stochastic ones.

The first, partial results show a significantly a lower model error compared to the previous method: the difference between simulation and real data is reduced by $11 \%$ considering the mean departure delay.

\section{Conclusions and outlook}

The importance of accuracy in railway operation planning is increasing, since it allows an optimal use of the existing infrastructures. Micro-simulation is the most accurate method to reproduce rail operations: therefore it is becoming essential in estimating a priori the impact of new timetable or infrastructure improvements.

In this paper a model that allows considering stop time variability in a more accurate way compared to the existing simulation models is introduced. The model requires a limited initial calibration effort, after which it can be applied to large networks simply using the track occupation data automatically collected by the Infrastructure Managers.

The model introduces some assumptions on the basis of extensive tests carried out in previous studies: these allow estimating the total dwell time as a function of multiple deterministic parameters and of a stochastic one that represents the number of passengers.

Thanks to this very simple structure, the model can easily be applied in practice to prepare the data sets for stochastic micro-simulation and therefore support timetable planning

\section{Further research}

While the precision of the model is generally satisfying regarding the estimation of the total dwell time to be used in simulation it does not allow any rough estimation of the total number of passengers boarding and alighting. Further investigation is therefore required to consider the distribution of passengers along a platform, possibly as a function of its physical layout and of the layout of the major origin/destination station on the line.

The model will be tested on larger networks, to improve the parameter set. This second calibration phase will lead to a more detailed estimation of parameters in three important cases: on long-distance services, where the interior could be co congested due to narrow corridors and heavy luggage, on short-distance trains on Mondays and Fridays, with a relevant number of weekly commuters and on overcrowded commuter trains, in which the stop time increases remarkably.

Finally, the approach will be adapted to high-frequency urban services, where the passengers waiting on a platform normally take the first train that stops leading to longer stop times on delayed trains. 


\section{References}

[1] Huerlimann D., Longo G., Medeossi G.: "Stochastic micro-simulation as a timetable robustness estimation tool" Proceedings of the "3nd International Seminar on Railway Operations Research (ISROR)”, Zurich 11-12 January 2009.

[2] de Fabris S., Longo G., Medeossi, G.: "An algorithm for the calibration of running time calculation on the basis of GPS data". In C.A. Brebbia, G.M. Carlomagno (Ed.) Computational Methods and Experimental Measurements XIV, WIT Press, Southampton, 2011. (pp. 577-586).

[3] Weidmann, U., Grundlagen zur Berechnung der Fahrgastwechselzeit, Institute Report No. 106, Institute for Transport Planning and Systems, Swiss Federal Institute of Technology Zurich, Zurich, 1995

[4] Tuna D., Fahrgastwechselzeit im Personefernverkehr, Thesis, TU Wien, 2008.

[5] Rueger B., Reisegepaeck im Eisenbahnfernverkehr, Dissertation, TU Wien, 2004.

[6] Transportation Research Board. Transit Capacity and Quality of Service Manual, 2nd Edition, 2003, TRCP Report 100.

[7] Heinz W., Passenger service times on trains - theory, measurements, and models, Licentiate Thesis, Royal Institute of Technology, Stockholm, 2003.

[8] Nash A., Weidmann U., Bollinger S., Luethi M., and Buchmueller S. (2006); Increasing schedule reliability on Zurich's S-Bahn through computer analysis and simulation, Transportation Research Record \#1955, Transportation Research Board, Washington D.C., pp. 17-25, 2007.

[9] Buchmueller S., Weidmann U., Nash A.: "Development of a dwell time calculation model for timetable planning". In Computers in Railways XI, WIT Press, Southampton, 2008. (pp. 525-534).

[10] Wang Z., Koutsopoulos H.N., Calibration of Urban Rail Simulation models: A methodology using SPSA Algorithm, Proceedings of the 2011 Winter Simulation Conference, Phoenix, 2011.

[11] Puong, A. "Dwell Time Model and Analysis for MBTA Red Line." Massachusetts Institute of Technology Research Memo, 2000. 